\title{
Hydrodynamics-based delivery of an interleukin-1 receptor II fusion gene ameliorates rat autoimmune myocarditis by inhibiting IL-1 and Th17 cell polarization
}

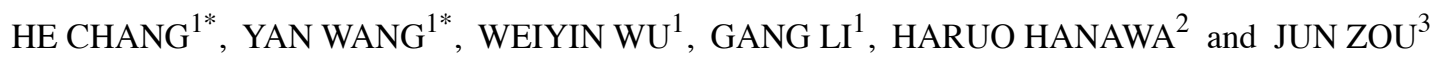 \\ ${ }^{1}$ Division of Cardiology, The Affiliated Zhongshan Hospital of Xiamen University, Xiamen Heart Center, Xiamen, Fujian, \\ P.R. China; ${ }^{2}$ Division of Cardiology, Niigata University Graduate School of Medical and Dental Sciences, Niigata, Japan; \\ ${ }^{3}$ Department of Pharmacology, School of Medical Sciences, Xiamen University, Xiamen, Fujian, P.R. China
}

Received November 11, 2012; Accepted January 11, 2013

DOI: 10.3892/ijmm.2013.1276

\begin{abstract}
Type II interleukin-1 receptor (IL-1RII) is a non-signaling decoy receptor that blocks the activity of interleukin-1 (IL-1), a pro-inflammatory cytokine involved in experimental autoimmune myocarditis (EAM). The aim of this study was to examine the effects of hydrodynamicsbased delivery of a recombinant plasmid encoding IL-1RII-Ig and to elucidate the role of IL-1RII in EAM rats. Rats were immunized on day 0 and injected with a recombinant plasmid encoding IL-1RII-Ig or pCAGGS-SP-Ig (control plasmid) on day 6. IL-1RII-Ig gene therapy effectively controlled EAM as indicated by a decreased heart weightto-body weight ratio, reduced areas of myocarditis, reduced expression of genes encoding atrial natriuretic peptide and brain natriuretic peptide in the heart, and improved cardiac function. IL-1RII-Ig significantly inhibited the expression of IL-1-related cytokines such as IL-1 $\beta$, prostaglandin E2 synthase, cyclooxygenase, and monocyte chemotactic protein-1 in EAM hearts. Furthermore, the effect of serum containing IL-1RII-Ig on the expression of immune-related genes in IL-1-stimulated splenocytes cultured from EAM rats was examined. The results showed that the expression of IL-6, transforming growth factor- $\beta$, retinoic acid-related orphan nuclear receptor (ROR $\gamma \mathrm{t}$ ) and IL-17, was significantly decreased upon exposure to serum containing IL-1RII-Ig. In conclusion, hydrodynamics-based delivery of a recombinant plasmid encoding IL-1RII-Ig effectively prevented progression of left ventricular remodeling and myocardial damage in
\end{abstract}

Correspondence to: Dr Jun Zou, Department of Pharmacology, School of Medical Sciences, Xiamen University, 168 Daxue, Xiamen, Fujian 361005, P.R. China

E-mail: zoujun@xmu.edu.cn

${ }^{*}$ Contributed equally

Key words: autoimmune myocarditis, type II interleukin-1 receptor, gene therapy
EAM rats. Moreover, IL-1RII may ameliorate experimental autoimmune myocarditis by blocking IL-1 and inhibiting production of the cytokines important for the polarization of T cells toward a Th17 phenotype.

\section{Introduction}

The importance of autoimmune-mediated mechanisms in the pathogenesis of myocarditis and dilated cardiomyopathy has been highlighted in recent studies $(1,2)$. Inflammatory cytokines activate the immune systems of patients or experimental animals via direct cytotoxic effects and by stimulating $\mathrm{T}$ and B cells. Rat experimental autoimmune myocarditis (EAM) resembles human giant cell myocarditis (3). Studies using EAM models have shown that cytokines such as interleukin (IL)-1 and tumor necrosis factor (TNF)- $\alpha$ play an important role in mediating cardiac dysfunction $(4,5)$.

IL-1 is an important cytokine and is mainly secreted by monocytes, macrophages, dendritic cells, B cells and NK cells (6-8). IL-1 comprises two distinct molecules, IL-1 $\alpha$ and IL-1 $\beta$, with high sequence homology and indistinguishable biological activity. Both IL- $1 \alpha$ and IL-1 $\beta$ bind to two primary receptors: type I IL-1 receptor (IL-1RI) and IL-1RII $(9,10)$. The binding of IL-1RI to IL-1 recruits IL-1Racp to form a heterodimeric receptor, which transmits a signal. In contrast, IL-1RII serves as a decoy receptor as it lacks an intracellular domain and does not transmit a signal (11). The function of IL-1 can be blocked by two physiological mechanisms: the IL-1 receptor antagonist (IL-1RA) associates with the IL-1RI to prevent the binding of IL-1 and restricts the recruitment of IL-1RAcp; IL-1RII, acting as a binding protein, binds to IL-1 but does not induce signal transduction as it lacks an intracellular domain. IL-1RII can also interact with IL-1RAcp (12-14). IL-1RA has been well studied over recent years and is now being used to treat a variety of diseases (15-18). However, there are few studies on IL-1RII, particularly with respect to cardiovascular disease. Furthermore, IL-17-producing cells (Th17 cells) have been identified, and studies have shown that Th17 cells play a central role in the pathogenesis of autoimmune diseases. The role of IL-1RII in driving Th17 commitment in EAM rats is unknown. 
Therefore, the aims of the present study were to examine the effect of hydrodynamics-based delivery $(19,20)$ of a plasmid encoding IL-1RII-Ig on EAM rats and to evaluate its possible mechanism.

\section{Materials and methods}

Animals. Eight-week-old male Lewis rats were purchased from Beijing Vital River Lab Animal Technology Co., Ltd. (Beijing, China) and maintained in our animal facility. All animals were treated in accordance with the Guidelines for Animal Experiments as laid out by the Laboratory Animal Center of Xiamen University, Fujian, China.

Induction of EAM. Cardiac myosin was a kind gift from the Division of Cardiology, Niigata University Graduate School of Medical and Dental Sciences, Niigata, Japan (3). For the induction of EAM, each rat was immunized with $0.2 \mathrm{ml}$ of an emulsion containing cardiac myosin and an equal volume of complete Freund's adjuvant supplemented with $10 \mathrm{mg} / \mathrm{ml}$ Mycobacterium tuberculosis H37RA (Difco Laboratories, Detroit, MI, USA) by a single s.c. injection in both footpads on day 0 .

Construction of the plasmid DNA for gene transfer. First, the plasmid vector, pCAGGS-IgGFc, containing SwaI and NotI restriction sites was prepared. The control plasmid, pCAGGSrat signal peptide (SP)-IgGFc, containing the SP region of the secretory leukocyte protease inhibitor, was constructed as previously described (18). To construct the pCAGGS-IL-1RIIIg plasmid, rat IL-1RII was amplified from EAM heart cDNA using the following primers, 5'-TTCATTTAAATGTTCAT CTTGCTTGTGTTA-3' and 5'-GCATCGCGGCCGCGGAA GAAACTTCTTTGA-3'. Rat SP was amplified from EAM heart cDNA using the following primers, 5'-GCCTTCACC ATGAAGTAAAG-3' and 5'-TGTATCCAACAGCATTTC CTTA-3'; and inserted into the vector, pCAGGS-IgGFc, using SwaI and NotI sites. E. coli JM109-competent cells were then transformed and the recombinant plasmids were isolated using an E.Z.N.A. Plasmid Giga kit (Omega Bio-Tek, USA).

Expression and distribution of IL-RII $m R N A$ in the rat. Twenty-five rats were divided into two groups: the control group $(n=7)$ receiving no immunization and the EAM group $(n=18)$ receiving immunization on day 0 . EAM rats were sacrificed on day $14(n=6)$, day $21(n=6)$, and day $28(n=6)$, respectively, and the organs (heart, liver, spleen and kidney) were harvested. To detect the biodistribution and the time course of IL-1RII expression, total RNA was isolated from the tissue. cDNA synthesis and real-time PCR of IL-1RII and GAPDH were carried out.

Plasmid DNA injection techniques. Thirty-two rats were randomly divided into two study groups and one control group, The normal group consisted of rats not immunized and not injected $(n=11)$. The 21 immunized rats were divided into two study groups. The IL-1RII group consisted of immunized rats injected with pCAGGS-IL-1RII-IgFc-(GLU)-tag $(n=11)$, and the SP group consisted of immunized rats injected with pCAGGS-SP-IgFc-(GLU)-tag $(n=10)$. Each immunized rat was injected with $800 \mu \mathrm{g}$ pCAGGS-IL-1RII-IgFc-(GLU)-tag or pCAGGS-SP-IgFc-(GLU)-tag dissolved in the appropriate volume of Ringer's solution via the tail vein within $15 \mathrm{sec}$ ( $\sim 80 \mathrm{ml} / \mathrm{kg}$ body weight) on day 6 . We used the method of hydrodynamics-based gene transfer, as this method is believed to be the most effective for facilitating elevated concentration levels.

Plasmid mRNA expression and chimeric GLU-tag protein measurement. Rats were injected with plasmid pCAGGS-IL1RII-IgFc-(GLU)-tag ( $n=3$ ) or pCAGGS-SP-IgFc-(GLU)-tag $(n=3)$ with immunization on day 6 and sacrificed on day 7. IL-RII-Ig or SP-Ig mRNA expression in liver tissues was examined to evaluate the efficiency of the hydrodynamics-based gene transfer (19). Total RNA was isolated from the livers, and cDNA was synthesized using M-MLV reverse transcriptase and oligo(dT). The RT products were amplified by PCR using TaqDNA polymerase (both were from Fermantas, USA). All PCR products were resolved using ethidium bromide-stained $2 \%$ agarose gels.

To measure plasma concentrations of IL-1RII-IgFcGLU-tag proteins during the treatment course, blood samples were taken on day $7(n=7)$, day $12(n=6)$, and day $17(n=7)$ respectively, after the hydrodynamics-based gene transfer on day 6. GLU concentrations were measured using a GLU RIA kit (Daiichi Radioisotope Laboratories, Tokyo, Japan). Chimeric protein concentrations were calculated using a GLU-tag.

Echocardiography. Echocardiography was performed on day 16 using a 14-MHz probe (Vivid 7; General Electric, USA). The left ventricular (LV) end-diastolic diameter (LVEDd), left ventricular end-systolic diameter (LVEDs), interventricular septal thickness (IVS), LV posterior wall thickness (LVPW), LV fractional shortening (LVFS), and the LV ejection fractions (LVEF) were calculated from the M-mode echocardiograms.

Evaluation of histopathology. All rats were sacrificed on day 17. The heart weight (without atria) and the body weight were measured, and the ratio of heart weight-to-body weight $(\mathrm{g} / \mathrm{g})$ was calculated. Hearts were fixed in $10 \%$ formalin, paraffin-embedded, and cut into $4-\mu \mathrm{m}$ transverse sections for Masson's trichrome staining. The area of the entire heart and the regions affected by myocarditis (regions showing myocardial necrosis, inflammatory cell infiltration and myocardial fibrosis) were calculated using image analysis software (Image-Pro Plus v. 6.0; Image-Pro, USA).

Relative expression of markers of heart failure and IL-1related cytokines in the heart. Total RNA was isolated from the apex of the heart on day 17. To evaluate the effects of gene therapy, the levels of two specific heart failure markers, atrial natriuretic peptide (ANP) and brain natriuretic peptide (BNP), were measured using quantitative real-time RT-PCR. IL-1-related cytokines in the heart tissues were also examined including IL-1 $\beta$, prostaglandin E2 synthases (PGEs), cyclooxygenase (COX-2), and monocyte chemotactic protein-1 (MCP-1) (Table I). After an initial denaturation step of $10 \mathrm{~min}$ at $95^{\circ} \mathrm{C}$, a 2 -step cycling procedure (denaturation at $95^{\circ} \mathrm{C}$ for $15 \mathrm{sec}$, annealing and extension at $60^{\circ} \mathrm{C}$ for $1 \mathrm{~min}$ ) was used 
Table I. Primers used for RT-PCR and quantitative RT-PCR.

\begin{tabular}{|c|c|c|}
\hline Gene & Sense primer & Antisense primer \\
\hline ANP & 5'-atggatttcaagaacctgctaga-3' & 5'-gctccaatcetgtcaatcctac-3' \\
\hline BNP & 5'-gatgattctgctcctgcttttc-3' & $5^{\prime}$-gccatttcctctgacttttctc- $3^{\prime}$ \\
\hline IL-1 $\beta$ & 5'-gctagtgtgtgatgttcccattag-3' & $5^{\prime}$-cttttccatcttcttctttgggta-3' \\
\hline PGEs & 5'-gtgatggagaacagccaggt-3' & 5'-gaggaccacgaggaaatgtatc-3' \\
\hline COX-2 & 5'-tgtgatattctcaaacaggagcat-3' & 5'-aaggaggatggagttgttgtagag-3' \\
\hline MCP-1 & 5'-ctgtctcagccagatgcagttaat-3' & 5'-tatgggtcaagttcacattcaaag-3' \\
\hline IL-6 & 5'-ccgagtagacctcatagtgacctt-3' & 5'-cctattgaaaatctgetctggtct-3' \\
\hline TGF- $\beta$ & 5'-tcagacattcgggaagcagtg-3' & 5'-attccgtctccttggttcagc-3' \\
\hline $\mathrm{ROR} \gamma \mathrm{t}$ & 5'-tctggaagctgtgggataga-3' & 5'-gaggagcctgtggagaaatac-3' \\
\hline IL-17 & 5'-tactcatccctcaaagttcagtgt-3' & 5'-ctcttgctggatgagaacagaat-3' \\
\hline GAPDH & 5'-atcaccatcttccaggagcga-3' & 5'-agccttctccatggtggtgga-3' \\
\hline
\end{tabular}

ANP, atrial natriuretic peptide; BNP, brain natriuretic peptide; IL-1 $\beta$, interleukin-1 $\beta$; PGEs, prostaglandin E2 synthase; COX-2, cyclooxygenase; MCP-1, monocyte chemotactic protein-1; TGF- $\beta$, transforming growth factor- $\beta$; ROR $\gamma$ t, retinoic acid-related orphan nuclear receptor.
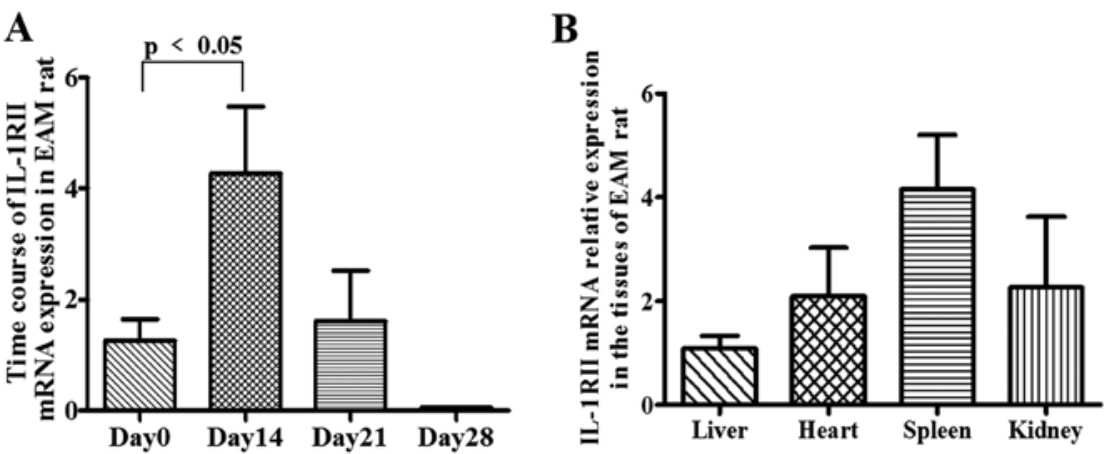

Figure 1. (A) Time course of IL-1RII expression in the hearts of EAM rats (days 0, 14, 21 and 28) as assessed by quantitative real-time PCR. (B) Biodistribution of IL-1RII in EAM rats as assessed by quantitative real-time PCR. EAM rats were sacrificed on day 14 and organs (heart, liver, spleen and kidney) were harvested.

for 40 cycles. Melting curve analysis was performed immediately after the amplification step. The levels of gene expression were normalized to that of GAPDH. Relative changes in the expression of these molecules was assessed by comparative analysis of the quantitative real-time PCR results using the $\Delta \Delta \mathrm{CT}$ method (21).

Spleen cell culture with serum containing IL-IRII-Ig. To prepare serum containing IL-1RII-Ig or SP-Ig for spleen cell culture, normal rats were injected with $800 \mu \mathrm{g}$ of pCAGGS-IL1RII-IgGFc or pCAGGS-SP-IgGFc dissolved in the appropriate volume of Ringer's solution ( $\sim 80 \mathrm{ml} / \mathrm{kg}$ body weight) via the tail vein within $15 \mathrm{sec}$, and the serum was collected after $24 \mathrm{~h}$. Spleens were obtained from EAM rats on day 17 and cultured at a density of $6 \times 10^{6}$ cells $/ \mathrm{ml}$ on $35 \mathrm{~mm}$-well dishes in $2 \mathrm{ml}$ of RPMI-1640 medium supplemented with $10 \%$ fetal calf serum (FCS). Shortly after culture, spleen cells were stimulated with rat IL-1 $\alpha$ (final concentration, $10 \mathrm{ng} / \mathrm{ml}$; PeproTech, UK) and $100 \mu \mathrm{l}$ of IL-1RII-Ig-GLU-tag-containing serum obtained from an IL-1RII-Ig-treated normal rat or the same amount of an Ig-GLU-tag-containing serum from an SP-Ig-treated normal rat (22).
Detection of Th17 cell-derived molecules using quantitative real-time $R T$-PCR. Spleen cells were collected after $24 \mathrm{~h}$ of culture at $37^{\circ} \mathrm{C}$, total RNA was isolated, and cDNA was synthesized as described above. The relative expression of IL-6, transforming growth factor- $\beta$ (TGF- $\beta$ ), retinoic acidrelated orphan nuclear receptor $(\mathrm{ROR} \gamma \mathrm{t})$ and IL-17 mRNA was measured by quantitative real-time RT-PCR (Table I). The $\Delta \Delta \mathrm{CT}$ method was used to quantitate gene expression.

Statistical analysis. Statistical analysis was performed using the unpaired Student's t-test or one-way ANOVA, and the Bonferroni multiple comparison test. Differences were considered significant at $\mathrm{P}<0.05$. The heart weight-to-body weight ratio, area of myocarditis, echocardiography and hemodynamic parameters, and the data obtained from quantitative RT-PCR were expressed as means \pm SEM.

\section{Results}

IL-1RII expression and distribution in EAM rats. The mRNA expression of IL-1RII in the hearts of EAM rats was increased, peaking at 4.269 \pm 1.208 -fold on day 14 (vs. day 0, 
Table II. Results of the echocardiograph.

\begin{tabular}{llll}
\hline & Normal $(\mathrm{n}=8)$ & SP-Ig $(\mathrm{n}=6)$ & IL-1RII-Ig $(\mathrm{n}=7)$ \\
\hline LVEDd (mm) & $6.410 \pm 0.1324$ & $6.981 \pm 0.1419^{\mathrm{a}}$ & $6.235 \pm 0.1396^{\mathrm{e}}$ \\
LVEDs (mm) & $3.990 \pm 0.1147$ & $4.680 \pm 0.1325^{\mathrm{b}}$ & $4.176 \pm 0.1038^{\mathrm{e}}$ \\
IVS (mm) & $1.314 \pm 0.04684$ & $1.378 \pm 0.05494$ & $1.282 \pm 0.04031$ \\
LVPW (mm) & $1.442 \pm 0.04693$ & $1.490 \pm 0.04161^{\mathrm{b}}$ & $1.353 \pm 0.03100^{\mathrm{d}}$ \\
LVFS (\%) & $39.31 \pm 0.9265$ & $29.23 \pm 1.429^{\mathrm{c}}$ & $36.67 \pm 1.415^{\mathrm{e}}$ \\
LVEF (\%) & $75.46 \pm 1.057$ & $61.83 \pm 2.149^{\mathrm{c}}$ & $70.11 \pm 1.519^{\mathrm{e}}$ \\
\hline
\end{tabular}

LVEDd, left ventricular end-diastolic internal diameter; LVESd, left ventricular end-systolic internal diameter; IVS, interventricular septal thickness; LVPW, left ventricular posterior wall thickness; LVFS, left ventricular fractional shortening; LVEF, left ventricular ejection fraction. Pericardial effusion was only detected in rats in the SP group. Results are expressed as the mean $\pm \mathrm{SEM}$. ${ }^{\mathrm{a}} \mathrm{P}<0.05,{ }^{\mathrm{b}} \mathrm{p}<0.01,{ }^{\mathrm{c}} \mathrm{p}<0.001 \mathrm{for}$ comparisons with rats in normal group. ${ }^{\mathrm{d}} \mathrm{p}<0.05,{ }^{\mathrm{e}} \mathrm{p}<0.01,{ }^{\mathrm{f}} \mathrm{p}<0.001$ for comparisons with rats in SP group.

A

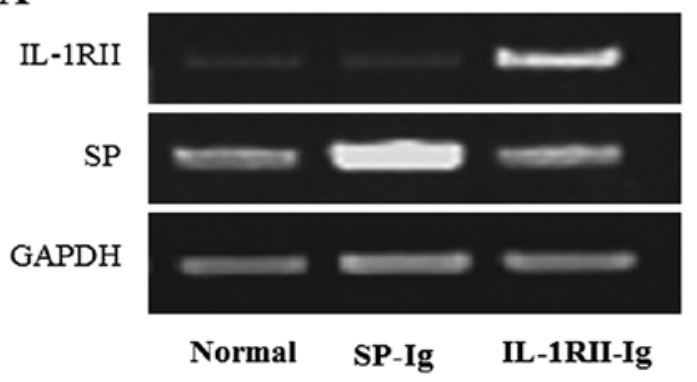

B

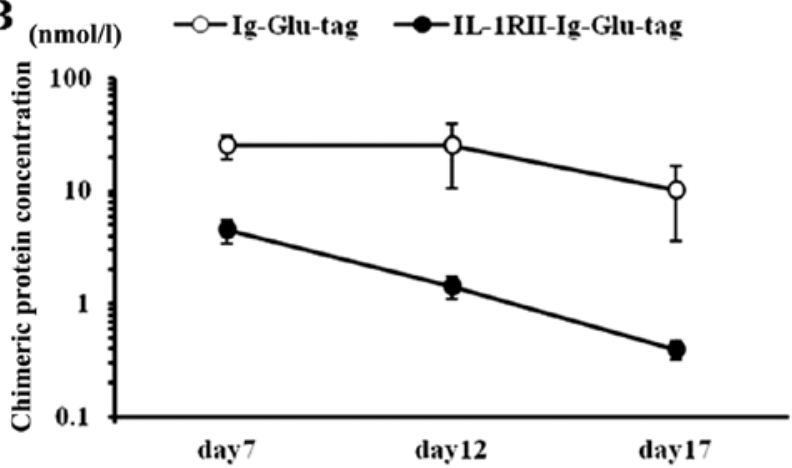

Figure 2. (A) IL-1RII-Ig or SP-Ig mRNA expression in liver tissues was examined. Rats were injected with plasmid on day 6 and sacrificed on day 7. Control rats were immunized but not injected with plasmid. SP rats were injected with plasmid pCAGGS-SP-IgGFc-(GLU)-tag. IL-1RII rats were injected with plasmid pCAGGS-IL-1RII-IgFc-(GLU)-tag). (B) Plasma levels of Ig-GLU-tag and IL-1RII-Ig-GLU-tag protein. The rats were injected with pCAGGS-rat SP-Ig-GLU-tag or pCAGGS-rat IL-1RII-Ig-GLU-tag on day 6.

$<0.05)$ and gradually decreasing to $1.61 \pm 0.9$-fold on day 21 and $0.056 \pm 0.009$-fold on day 28 (Fig. 1A). The distribution of IL-1RII in the liver, heart, spleen and kidney of EAM rats showed on day 14 that IL-1RII gene expression was highest in the spleen and lowest in the liver (Fig. 1B).

Plasma IL-1RII-Ig-GLU-tag protein levels. The expression of injected plasmid IL-1RII or SP in the liver was examined using RT-PCR. The results showed that expression of IL-1RII or SP significantly increased $24 \mathrm{~h}$ after injection of the recombinant plasmid IL-1RII or SP (Fig. 2A). During the course of treatment, plasma IL-1RII-Ig-GLU-tag protein levels in rats injected with pCAGGS-IL-1RII-Ig on day 6 increased to $4.49 \pm 1.1 \mathrm{nmol} / 1$ (mean $\pm \mathrm{SEM}$ ) on day 7 and gradually decreased on days 12 and 17 to $1.43 \pm 0.33$ and $0.4 \pm 0.08 \mathrm{nmol} / 1$, respectively. Plasma Ig-GLU-tag protein levels in the pCAGGS-SP-Ig control rats increased to $25.28 \pm 5.97 \mathrm{nmol} / 1$ on day 7 and decreased on days 12 and 17 to $25.02 \pm 14.33$ and $10.18 \pm 6.55 \mathrm{nmol} / 1$, respectively (Fig. 2B). It has been reported that IL-1RII $(0.5$ to $1.5 \mathrm{nmol} / 1$ and 0.007 to $0.014 \mathrm{nmol} / \mathrm{l})$ suppresses the production of PGEs from human chondrocytes stimulated by IL-1 $\beta$ in vitro (23). These results indicated that continuous effective delivery of the IL-1RII-Ig protein for
$>17$ days can be achieved in rats by hydrodynamics-based gene transfer.

Echocardiography and hemodynamic parameters. The LVEDd, LVEDs and LVPW values in the IL-1RII-Ig group were significantly smaller than these values in the SP-Ig group. The LV fractional shortening (LVFS\%) and LV ejection fraction (LVEF\%) in the IL-1RII-Ig group were significantly higher than those in the SP-Ig group (Table II). This indicated that the degree of heart failure in the IL-1RII group was relieved while that in the SP-Ig group was not.

Effect of in vivo treatment with plasmid DNA encoding the IL-IRII-Ig gene. The heart-to-body weight ratio in the IL-1RII-Ig group was significantly lower than that in the SP-Ig group $(0.36 \pm 0.01$ vs. $0.44 \pm 0.02 \% ; p=0.0002)$ (Fig. 3A). Many inflammatory cells and fibroblasts had infiltrated the SP-Ig group hearts, but fewer inflammatory cells were observed in the hearts of IL-1RII-Ig rats. The area of myocarditis in the IL-1RII-Ig group was significantly smaller when compered with that in the SP-Ig group $(18.40 \pm 1.20$ vs. $37.51 \pm 0.79 \%$; $\mathrm{p}<0.0001$ ) (Figs. 3B and 4). The relative expression of ANP mRNA was significantly lower in heart tissues from the 

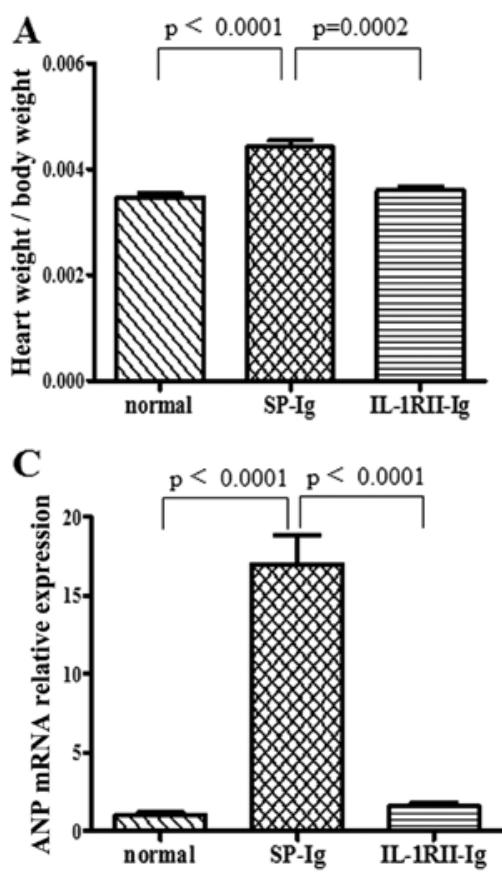
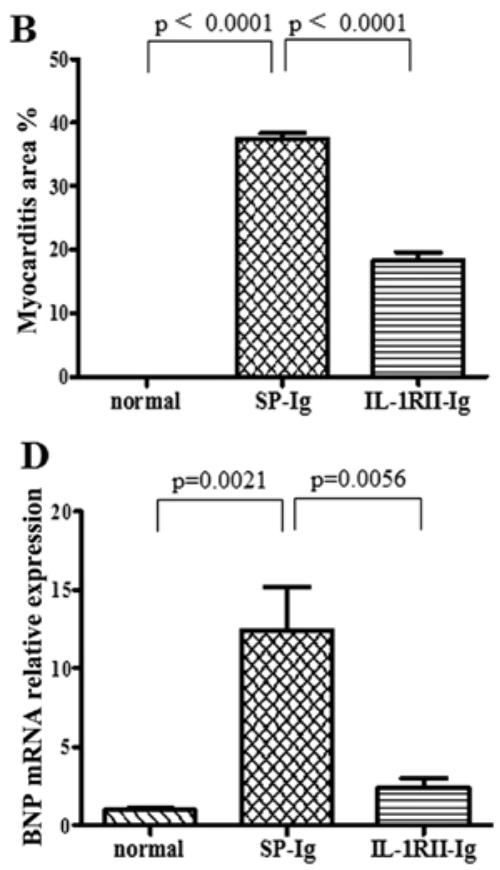

Figure 3. (A) Ratio of heart-to-body weight. (B) Area of myocarditis in the hearts of normal, SP-Ig and IL-1RII rats calculated using a color image analyzer. (C) Relative expression of ANP mRNA in hearts. (D) The relative expression of BNP mRNA in hearts. Normal rats were neither immunized nor injected with plasmid. SP-Ig rats were immunized on day 0 and injected with plasmid pCAGGS-SP-IgGFc-(GLU)-tag on day 6; IL-1RII-Ig rats were immunized on day 0 and injected with plasmid pCAGGS-IL-1RII-IgFc-(GLU)-tag on day 6. Statistical analysis was performed using one-way ANOVA or non-paired t-tests.

A

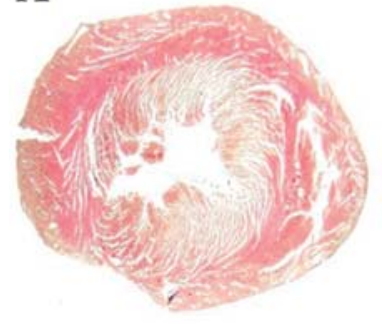

B

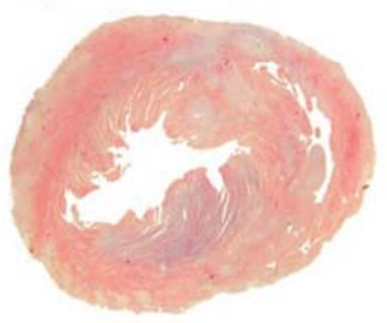

C

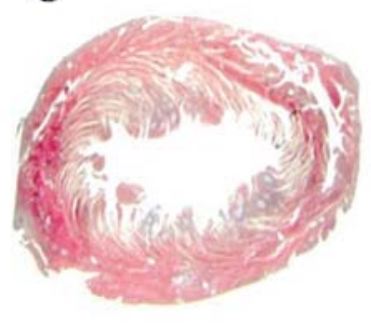

Figure 4. Histological examination of transverse sections showing ventricles stained with Masson's trichrome stain. (A) Transverse sections of a normal heart. (B) Transverse sections of a heart in the SP-Ig group. (C) Transverse section of a heart in the IL-1RII group. Images are representative of each group.

IL-1RII-Ig group when compard with that in the SP-Ig group $(1.58 \pm 0.22$ vs. $16.99 \pm 1.84 ; \mathrm{p}<0.0001)$ (Fig. 3C). Expression of BNP mRNA was also significantly lower in the IL-1RII-Ig group when compared with that in the SP-Ig group $(2.41 \pm 0.58$ vs. 12.40 $\pm 2.78 ; \mathrm{p}=0.0056$ ) (Fig. 3D).

Expression of IL-1-related cytokines in the EAM hearts. The mRNA expression of IL-1 $\beta$, PGEs, COX-2 and MCP- 1 on day 17 in EAM hearts was detected. The results showed that the expression of the following cytokines was significantly inhibited in the IL-1RII-Ig group: IL-1 $\beta(0.78 \pm 0.05$ vs. SP $9.45 \pm 0.18, \mathrm{p}<0.0001)$; PGEs $(0.44 \pm 0.14$ vs. SP $41.97 \pm 6.91$, $\mathrm{p}=0.0003)$; COX-2 (0.46 \pm 0.17 vs. SP 2.478 $\pm 0.13, \mathrm{p}<0.0001)$; MCP-1 (0.94 \pm 0.21 vs. SP 45.80 \pm 2.10, p<0.0001) (Fig. 5).

Expression of Th17 cell-derived molecules in splenocytes cultured with serum containing IL-IRII-Ig. Compared with SP-Ig-containing serum, IL-1RII-Ig-containing serum had significantly reduced expression of the Th17 cell-derived molecules including IL-6 $(0.92 \pm 0.07$ vs. $3.14 \pm 0.35 ; \mathrm{p}=0.0003)$, TGF- $\beta(1.86 \pm 0.12$ vs. $3.49 \pm 0.24, p=0.0003), R O R \gamma t(0.55 \pm 0.06$ vs. $17.08 \pm 2.07, \mathrm{p}<0.0001)$ and IL-17 (4.18 \pm 1.68 vs. $39.96 \pm 7.66$, $\mathrm{p}=0.0031$ ) at the mRNA level in cultivated splenocytes (Fig. 6).

\section{Discussion}

The aim of the present study was to examine the effect of hydrodynamics-based delivery of a recombinant plasmid encoding IL-1RII-Ig on EAM. The method used to deliver the naked plasmid was initially reported by Liu et al (24) and Zhang et al (25). These studies showed that extremely high levels of foreign gene expression in hepatocytes were achieved by hydrodynamics-based delivery; foreign proteins were expressed in the liver and delivered to other organs such as the heart and kidney via the circulation $(26,27)$. Furthermore, the fusion of cytokines with Ig-Fc segments offers advantages 

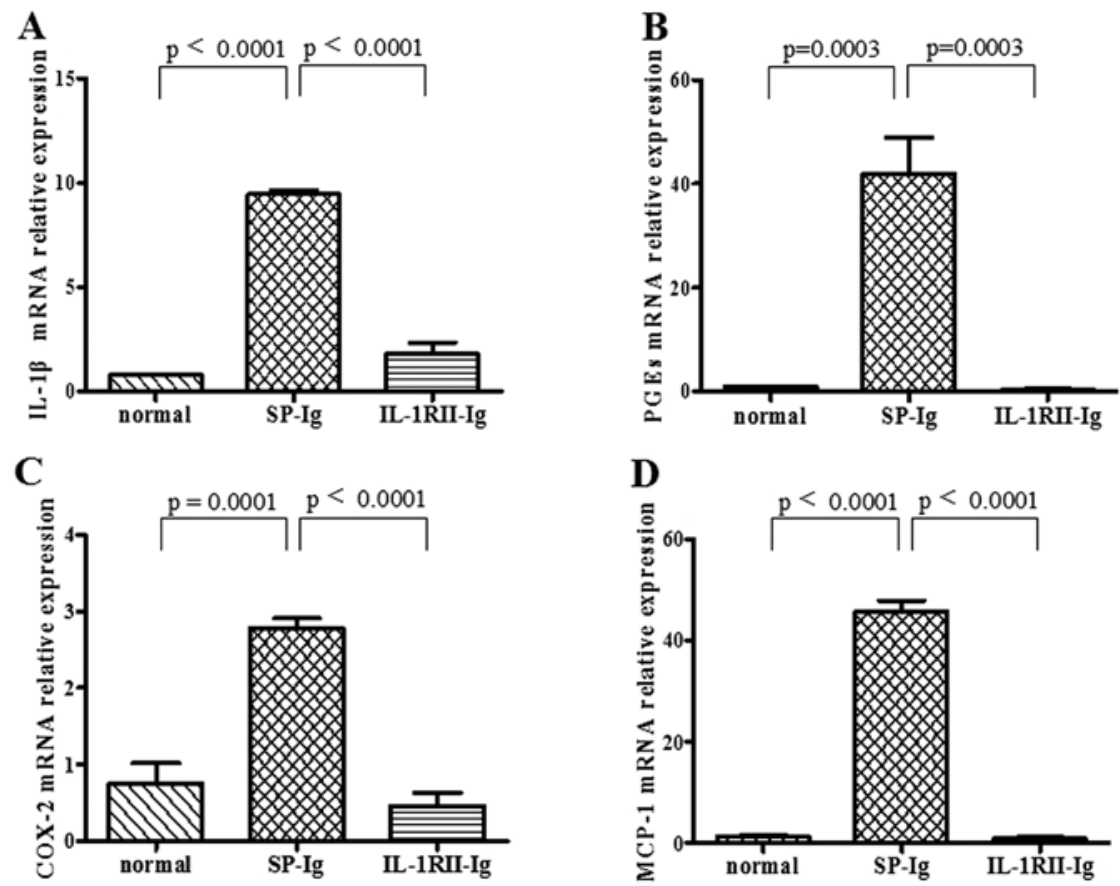

Figure 5. Relative expression of (A) IL-1 1 , (B) PGEs, (C) COX-2 and (D) MCP-1 mRNA in hearts from the normal, SP-Ig and IL-1RII-Ig groups as assessed by quantitative real-time PCR.
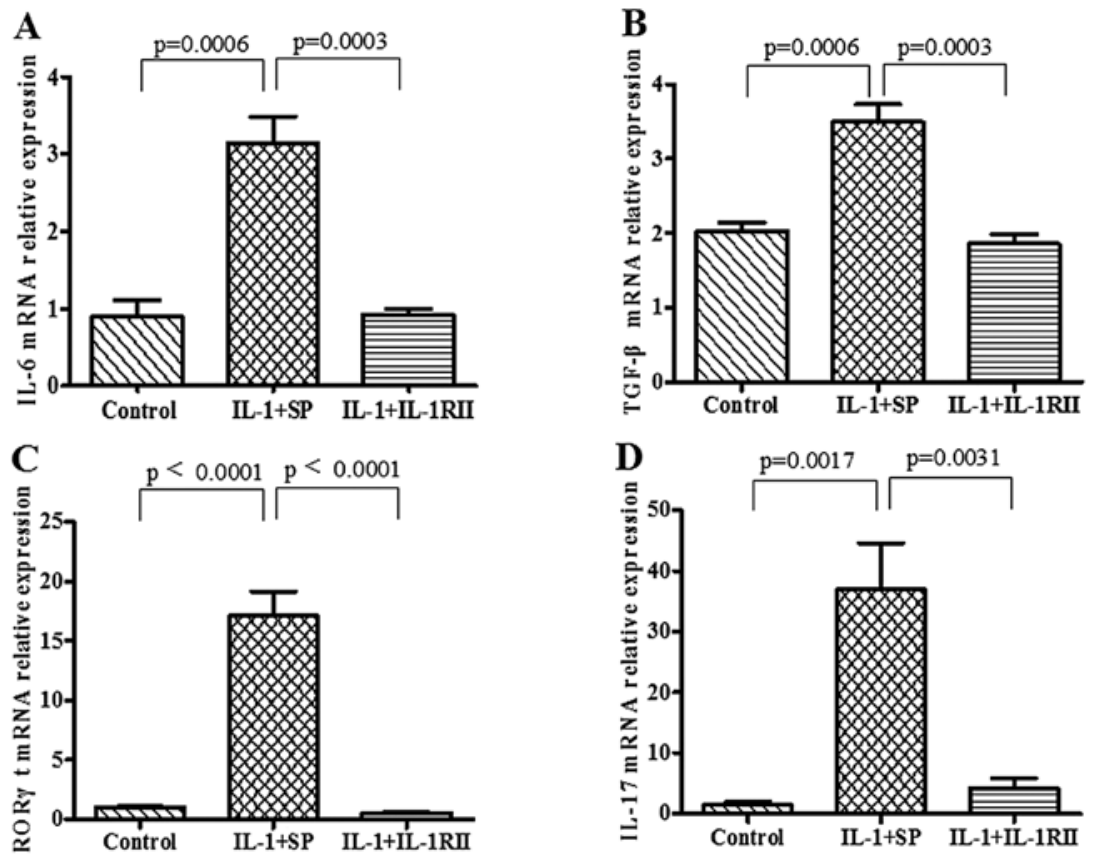

Figure 6. Relative expression of (A) IL-6, (B) TGF- $\beta$, (C) ROR $\gamma$ t and (D) IL-17 mRNA in splenocyte cultures derived from EAM rats. Control, no IL-1 $\alpha$ or serum: IL-1+SP: IL-1 $\alpha(10 \mathrm{ng} / \mathrm{ml})$ and $100 \mu 1$ serum obtained from a normal rat injected with pCAGGS-SP-IgGFc. IL-1+IL-1RII: IL-1 $\alpha$ (10 ng/ml) and $100 \mu 1$ serum obtained from a normal rat injected with pCAGGS-IL-1RII-Ig. Error bars represent SEM. Statistical analysis was performed using a one-way ANOVA or a non-paired t-test (control vs. IL-1+SP vs. IL-1+IL-1RII).

over native cytokines, such as an extended half-life in the circulation, characteristic of Igs, and a higher avidity for the ligand $(28,29)$.

In the present study, exogenous IL-1RII (expressed in vivo) appeared to inhibit IL-1-induced immune responses. IL-1, particularly IL-1 $\beta$, plays an important role in the pathogenesis of EAM which is an animal model for $\mathrm{CD} 4^{+} \mathrm{T}$ cell-mediated inflammatory heart disease (2). IL-1 expression is rapidly upregulated during the early phase of EAM, and can promote the proliferation and survival of naive T cells. IL-1 binds to IL-1RI and recruits the IL-1 receptor accessory protein (IL-1RAcp) to form a heterodimeric receptor, which is required for signal transduction (30). Therapies that block IL-1 signaling have been proposed over the past decade. Studies on IL-1RA 
have shown that it can effectively bind to IL-1RI and inhibit the activity of either IL- $1 \alpha$ or IL- $\beta$ (16-18). Similar to IL-1RA, IL-1RII serves as a decoy receptor and can inhibit IL-1 activity by binding to it without inducing signal transduction. IL-1RII has high affinity for IL-1, but a much lower affinity for IL-1RA, which allows IL-1RII to act as an IL-1 inhibitor. In addition, IL-1RAcP can be recruited to the IL-1RII-IL-1 complex so that the decoy receptor can sequester the accessory receptor and prevent it from participating in IL-1 signaling mediated by IL-1RI. This sequestration of IL-1RAcp greatly increases the inhibitory potency of IL-1RII (31). Recent studies showed that increased IL-1RII levels can neutralize IL-1 $\beta$ and effectively ameliorate autoimmune disease. Consistent with this, our data showed that the plasmid encoding IL-1RII-Ig effectively prevents the progression of LV remodeling and myocardial damage in EAM rats.

The mechanism underlying the etiology of autoimmune myocarditis remains unknown, but modulating Th1/Th2 balance appears to have a beneficial effect on the disease. During the past decade, studies have shown that treatments can alter the helper $\mathrm{T}$ cell balance: i.e. decrease expression of Th1 cytokines and increase expression of Th2 cytokines. It has been proven that blocking the activity of IL-1 may result in a reduction in Th1 cytokines and IL-1-induced production of PGEs, COX-2 and MCP-1 (18). This, again, is consistent with our results.

Recent findings indicate that Th17 cells appear to play a significant role in the progression of autoimmune diseases, and that IL-1 may induce the polarization of T cells toward a Th17 phenotype; however, the relationship between IL-1 signaling and Th17 cells in EAM rats has not been elucidated. IL-1, along with other cytokines, can drive Th17 cell polarization. Chung et al (32) reported a critical role of IL-1 in Th17 cell differentiation, and this pathway may serve as a unique target for Th17 cell-mediated immunopathology. Veldhoen and colleagues indicated that IL-1 can increase the number of Th17 cells generated in vitro in the presence of IL- 6 plus TGF- $\beta$ (33). ROR $\gamma$ t is the key transcription factor that orchestrates the differentiation of Th17 cells. ROR $\gamma$ t-deficient $\mathrm{CD}^{+}{ }^{+} \mathrm{T}$ cells do not produce IL-17 in response to TGF- $\beta$ and IL-6 (34). Our results showed that, in vitro, serum containing IL-1RII-Ig inhibited the expression of IL-6, TGF- $\beta$ and ROR $\gamma \mathrm{t}$ during Th17 cell polarization, which may suppress the secretion of IL-17. However, the association between blocking IL-1 signaling and a reduction in the level of Th17 cell-related cytokines remains elusive and further studies are needed.

In conclusion, the results of the present study suggest that hydrodynamics-based delivery of a recombinant plasmid encoding IL-1RII-Ig ameliorates EAM in rats. The possible mechanism may be through blocking IL-1 and inhibiting production of the cytokines critical for the polarization of T cells toward a Th17 phenotype. Our future studies will focus on identifying therapy targets based on this mechanism, which will aid in the prevention of autoimmune myocarditis.

\section{Acknowledgements}

This study was supported by the National Natural Science Foundation of China (grant no. 81270294) and the Natural Science Foundation of Fujian Province (grant no. 2012J01415).

\section{References}

1. Blauwet LA and Cooper LT: Myocarditis. Prog Cardiovase Dis 52: 274-288, 2010

2. Leuschner F, Katus HA and Kaya Z: Autoimmune myocarditis: past, present and future. J Autoimmun 33: 282-289, 2009.

3. Kodama M, Matsumoto Y, Fujiwara M, Masani F, Izumi T and Shibata A: A novel experimental model of giant cell myocarditis induced in rats by immunization with cardiac myosin fraction. Clin Immunol Immunopathol 57: 250-262, 1990.

4. Arend WP and Dayer JM: Inhibition of the production and effects of interleukin-1 and tumor necrosis factor alpha in rheumatoid arthritis. Arthritis Rheum 38: 151-160, 1995.

5. Vicenová B, Vopálenský V, Burýsek L and Pospísek M: Emerging role of Interleukin-1 in cardiovascular diseases. Physiol Res 58: 481-498, 2009.

6. Scala G, Allavena P, Djeu JY, Kasahara T, Ortaldo JR, Herberman RB and Oppenheim JJ: Human large granular lymphocytes are potent producers of interleukin-1. Nature 309: 56-59, 1984.

7. March CJ, Mosley B, Larsen A, et al: Cloning sequence and expression of two distinct human interleukin-1 complementary DNAs. Nature 315: 641-647, 1985.

8. Dinarello CA, Donath MY and Mandrup-Poulsen T: Role of IL-1beta in type 2 diabetes. Curr Opin Endocrinol Diabetes Obes 17: 314-321, 2010.

9. Bujak M and Frangogiannis NG: The role of IL-1 in the pathogenesis of heart disease. Arch Immunol Ther Exp 57: 165-176, 2009.

10. Dinarello CA: IL-1: discoveries, controversies and future directions. Eur J Immunol 40: 599-606, 2010.

11. Colotta F, Re F, Muzio M, et al: Interleukin-1 type II receptor: a decoy target for IL-1 that is regulated by IL-4. Science 261: $472-475,1993$.

12. Greenfeder SA, Nunes P, Kwee L, Labow M, Chizzonite RA and $\mathrm{Ju}$ G: Molecular cloning and characterization of a second subunit of the interleukin 1 receptor complex. J Biol Chem 270: 13757-13765, 1995.

13. Smith DE, Hanna R, Della Friend, et al: The soluble form of IL-1 receptor accessory protein enhances the ability of soluble type II IL-1 receptor to inhibit IL-1 action. Immunity 18: 87-96, 2003.

14. Sims JE and Smith DE: The IL-1 family: regulators of immunity. Nat Rev Immunol 10: 89-102, 2010.

15. Larsen CM, Faulenbach M, Vaag A, et al: Interleukin-1-receptor antagonist in type 2 diabetes mellitus. N Engl J Med 356: 1517-1526, 2007.

16. El-Osta H, Janku F and Kurzrock R: Successful treatment of Castle-man's disease with interleukin-1 receptor antagonist (Anakinra). Mol Cancer Ther 9: 1485-1488, 2010.

17. Mertens M and Singh JA: Anakinra for rheumatoid arthritis: a systematic review. J Rheumatol 36: 1118-1125, 2009.

18. Liu H, Hanawa H, Yoshida T, et al: Effect of hydrodynamicsbased gene delivery of plasmid DNA encoding interleukin-1 receptor antagonist-Ig for treatment of rat autoimmune myocarditis: possible mechanism for lymphocytes and noncardiac cells. Circulation 111: 1593-1600, 2005.

19. Maruyama H, Higuchi N, Nishikawa Y, et al: High-level expression of naked DNA delivered to rat liver via tail vein injection. J Gene Med 4: 333-341, 2002.

20. Abe S, Hanawa H, Hayashi M, et al: Prevention of experimental autoimmune myocarditis by hydrodynamics-based naked plasmid DNA encoding CTLA4-Ig gene delivery. J Card Fail 11: 557-564, 2005.

21. Livak KJ and Schmittgen TD: Analysis of relative gene expression data using real-time quantitative PCR and the 2(-Delta Delta C(T)) method. Methods 25: 402-408, 2001.

22. Chang H, Hanawa H, Yoshida T, et al: Alteration of IL-17 related protein expressions in experimental autoimmune myocarditis and inhibition of IL-17 by IL-10-Ig fusion gene transfer. Circ J 72: 813-819, 2008.

23. Attur MG, Dave M, Cipolletta C, et al: Reversal of autocrine and paracrine effects of interleukin 1 (IL-1) in human arthritis by type II IL-1 decoy receptor. Potential for pharmacological intervention. J Biol Chem 275: 40307-40315, 2000.

24. Liu F, Song Y and Liu D: Hydrodynamics-based transfection in animals by systemic administration of plasmid DNA. Gene Ther 6: 1258-1266, 1999. 
25. Zhang G, Budker V and Wolff JA: High levels of foreign gene expression in hepatocytes after tail vein injections of naked plasmid DNA. Hum Gene Ther 10: 1735-1737, 1999.

26. Chang $\mathrm{H}$, Hanawa $\mathrm{H}$, Liu $\mathrm{H}$, et al: Hydrodynamic-based delivery of an interleukin-22-Ig fusion gene ameliorates experimental autoimmune myocarditis in rats. J Immunol 177: 3635-3643, 2006.

27. Higuchi N, Maruyama H, Kuroda T, et al: Hydrodynamics-based delivery of the viral interleukin-10 gene suppresses experimental crescentic glomerulonephritis in Wistar-Kyoto rats. Gene Ther 10: 1297-1310, 2003.

28. Jiang J, Yamato E and Miyazaki J: Sustained expression of Fc-fusion cytokine following in vivo electroporation and mouse strain differences in expression levels. J Biochem 133: 423-427, 2003.

29. Elnaggar R, Hanawa $\mathrm{H}, \mathrm{Liu} \mathrm{H}$, et al: The effect of hydrodynamicsbased delivery of an IL-13-Ig fusion gene for experimental autoimmune myocarditis in rats and its possible mechanism. Eur J Immunol 35: 1995-2005, 2005.
30. Dinarello CA: Immunological and inflammatory functions of the interleukin 1 family. Annu Rev Immunol 27: 519-550, 2009.

31. Boraschi D and Tagliabue A: The interleukin-1 receptor family. Vitam Horm 74: 229-254, 2006.

32. Chung Y, Chang SH, Martinez GJ, et al: Critical regulation of early Th17 cell differentiation by interleukin-1 signaling. Immunity 30: 576-587, 2009.

33. Veldhoen M, Hocking RJ, Atkins CJ, Locksley RM and Stockinger B: TGFbeta in the context of an inflammatory cytokine milieu supports de novo differentiation of IL-17producing T cells. Immunity 24: 179-189, 2006.

34. Aranami T and Yamamura T: Th17 cells and autoimmune encephalomyelitis (EAE/MS). Allergol Int 57: 115-120, 2008. 